Technical Note

\title{
The impact and freezing processes of a water droplet on a cold surface with different inclined angles
}

\author{
Zheyan Jin $^{\mathrm{a}, \mathrm{b}, \mathrm{c}, *}$, Huanhuan Zhang ${ }^{\mathrm{b}}$, Zhigang Yang ${ }^{\mathrm{a}}$ \\ a Shanghai Key Lab of Vehicle Aerodynamics and Vehicle Thermal Management Systems, Shanghai 201804, China \\ ${ }^{\mathrm{b}}$ School of Aerospace Engineering and Applied Mechanics, Tongji University, Shanghai 200092, China \\ 'State Key Laboratory for Nonlinear Mechanics, Beijing 100190, China
}

\section{A R T I C L E I N F O}

\section{Article history:}

Received 30 May 2016

Received in revised form 7 August 2016

Accepted 8 August 2016

Available online 13 August 2016

\section{Keywords:}

Impact

Freezing

Droplet

Cold surface

Inclined angle

\begin{abstract}
A B S T R A C T
The present study experimentally investigated the impact process of a water droplet on a cold surface with different inclined angles. The inclined angle of the surface was changed from $0.0^{\circ}$ to $60.0^{\circ}$, while the surface temperature was varied from room temperature $21.0^{\circ} \mathrm{C}$ to $-15.0{ }^{\circ} \mathrm{C}$. During the impact process of the water droplet, the inclined angle of the surface was found to have an apparent influence on the spreading time and displacements of the foremost and rearmost points. Besides, once the inclined angle of the surface was high enough (e.g., $\alpha=60.0^{\circ}$ ), the water droplet could not keep as a single droplet all the time during its impact process. It split into two smaller droplets and produced satellite droplets at the same time. In addition, during the freezing process of the water droplet, the inclined angle of the surface could affect the final geometry of the ice bead.
\end{abstract}

(C) 2016 Elsevier Ltd. All rights reserved.

\section{Introduction}

Ice accretion on cold surfaces poses hazards to a variety of applications, such as wind turbines [1], aircraft [2], and power transmission lines [3], etc. In general, the ice accretion on these applications involves the impact and freezing processes of water droplets on an inclined cold solid surface. Understanding such impact and freezing processes under different conditions would be very desirable for researchers to develop more efficient antiicing or de-icing methods.

Over the years, many studies have been carried out on the impact process of a water droplet on an inclined surface. For example, Kang and Lee [4] studied the dynamic behavior of a water droplet impinging upon a heated surface. The results showed that droplet behavior after impact was greatly influenced by the normal momentum of the impinging droplet. Šikalo et al. [5] investigated drop impacts onto dry walls and liquid films at low impact angles and low normal Weber numbers. Šikalo and Ganić [6] observed the different characteristic features of the impact processes of droplets on different surfaces. The outcome of an impact was found to depend on the droplet properties and the impacted surface. Cui et al. [7] studied the impact processes of droplets on an inclined

\footnotetext{
* Corresponding author at: School of Aerospace Engineering and Applied Mechanics, Tongji University, Shanghai 200092, China.

E-mail address: zheyanjin@tongji.edu.cn (Z. Jin).
}

surface at different impact angles and Weber numbers. The surface temperature was kept at room temperature. Mishchenko et al. [8] performed an experimental and theoretical study of dynamic droplet freezing on structured surfaces. Ueda et al. [9] reported the trajectory of a water droplet of volume $0.4 \mu \mathrm{L}$ impacting and bouncing on the hydrophobic surface $\left(143^{\circ}\right)$ inclined with $12^{\circ}$. $\mathrm{Xu}$ et al. [10] observed the impact process of a water droplet on a Teflon plate and a stainless-steel plate, the inclined angle of which was $30^{\circ}$. Gilet and Bush [11] performed an experimental investigation of droplets impacting an inclined solid substrate coated with a thin layer of high viscosity liquid. Recently, Wang et al. [12] prepared four aluminum surfaces with wettability varied from superhydrophilic to superhydrophobic by combining an etching and a coating process. Quan et al. [13] applied a latticeBoltzmann with interparticle potential model for two-phase flow to simulate the dynamic behavior of droplet impact onto horizontal and inclined solid surfaces. Liang et al. [14] observed the spreading and splashing processes during a liquid drop impact on an inclined wetted surface. The results showed that both surface tension and viscosity could largely affect the spreading and splashing behaviors. More recently, Ramachandran et al. [15] studied the dynamics of droplets impacting the surface of hydrophobic concrete. Jin et al. [16] investigated the effects of droplet size and surface temperature on the impact, freezing, and melting processes of a water droplet on an inclined red copper surface. Besides, Jin et al. [17] investigated the impact and freezing processes of a water 
droplet on different inclined cold surfaces. The materials of the surfaces included red copper, quartz glass, carbon fiber reinforced composite, and glass fiber reinforced composite.

Even though extensive researches have been performed on the impact process of water droplets on an inclined surface in previous studies [4-17], to the authors' best knowledge, the detailed impact process of a water droplet on a cold surface with different inclined angles has not yet been experimentally investigated. In this study, the impact and freezing processes of a water droplet on a cold surface with different inclined angles were carefully measured. During the experiment, the water droplet was released from a droplet generator and impinged the inclined surfaces from a certain distance. A parameter study of the surface temperature was also carried out. The present study is aimed to improve our understanding about the microphysical processes pertinent to the droplet impact and icing phenomena.

\section{Experimental}

\subsection{Experimental setup}

The current experimental setup has been described in our previous studies $[16,17]$. The interested readers are referred to Refs. $[16,17]$ for details. It should be noted that a quartz glass test plate
$(50.0 \mathrm{~mm} \mathrm{~L} \times 50.0 \mathrm{~mm} \mathrm{~W} \times 1.5 \mathrm{~mm} \mathrm{H})$ was used in the present study. Besides, a high-speed camera (FR180, Norpix) was positioned perpendicularly to the original high speed camera (Dimax HD, PCO).

\subsection{Droplet characteristics}

The vertical distance between the test plate and the tip of the droplet generator was $20.0 \mathrm{~cm}$. the equivalent diameter of the droplet, $D_{0}$, was measured to be $2.92 \mathrm{~mm}$. Besides, the velocity of the water droplet right before it impinged the test plate, $U_{0}$, was calculated to be $1.967 \mathrm{~m} / \mathrm{s}$. The uncertainty of the terminal velocity was estimated to be within $0.025 \mathrm{~m} / \mathrm{s}$. Some dimensionless numbers of the droplet, such as Weber number, Bond number, Ohnesorge number, and Reynolds number, was estimated to be 155.32 , $1.1488,2.129 \times 10^{-3}$, and 5854.88 , respectively.

\subsection{Test plate properties}

Since the impact and freezing processes of the water droplet involve both wettability and heat conduction capability of the quartz glass test plate, the contact angle of a sessile water droplet $(V=2.0 \mu \mathrm{L})$ on the test plate, the surface roughness ( $\mathrm{Ra})$, and the

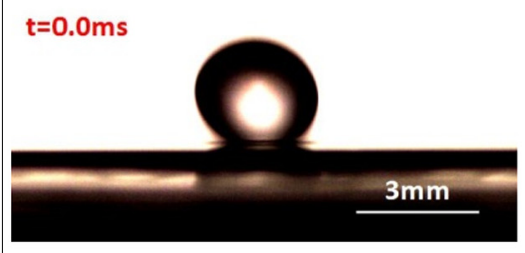

(a)

$$
\mathrm{t}=1.5 \mathrm{~ms}
$$

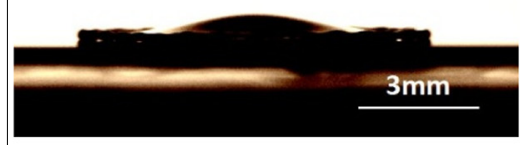

(d)

$$
t=14.5 \mathrm{~ms}
$$

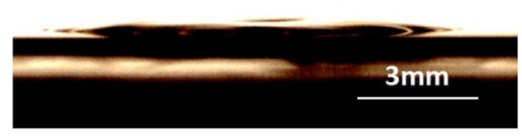

(g)

$$
\mathrm{t}=150.0 \mathrm{~ms}
$$

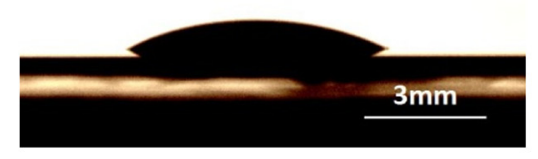

(j)

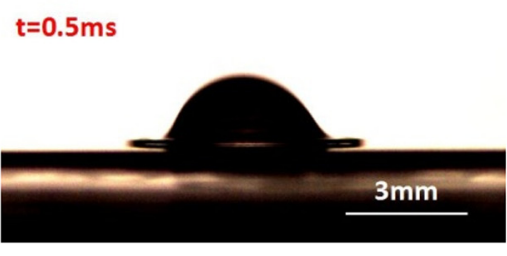

(b)

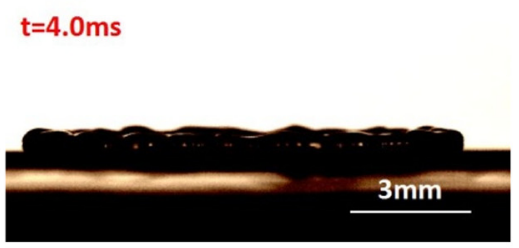

(e)

$\mathrm{t}=25.0 \mathrm{~ms}$

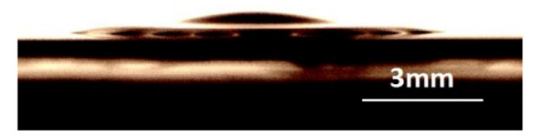

(h)

$$
\mathrm{t}=550.0 \mathrm{~ms}
$$

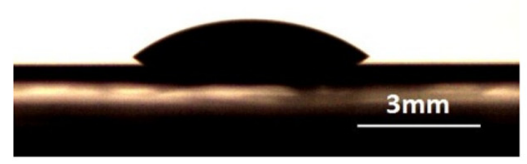

(k) $\mathrm{t}=1.0 \mathrm{~ms}$

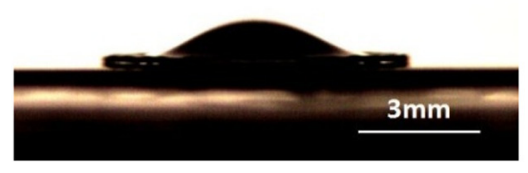

(c)

$\mathrm{t}=10.0 \mathrm{~ms}$

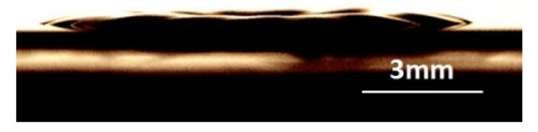

(f)

$\mathrm{t}=50.0 \mathrm{~ms}$

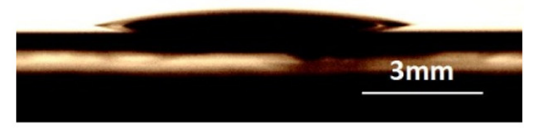

(i)

$\mathrm{t}=1100.0 \mathrm{~ms}$

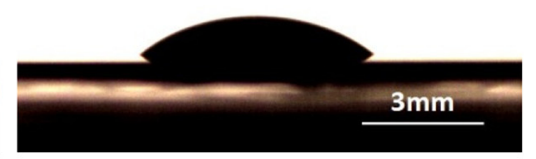

(1)

Fig. 1. Sequence of images of the water droplet impact process at an inclined angle of $0.0^{\circ}\left(T_{w}=-10.0^{\circ} \mathrm{C}\right)$. 
thermal conductivity of the test plate were measured to be $50.1^{\circ}$, $0.04 \mu \mathrm{m}$, and $1.234 \mathrm{~W} /(\mathrm{m} \cdot \mathrm{K})$, respectively.

\section{Results and discussions}

During the experiment, the relative humidity and temperature of the air in the laboratory were kept at $45.0 \pm 4.0 \%$ and $21.0 \pm 0.5^{\circ} \mathrm{C}$, respectively. The surface temperature $\left(T_{w}\right)$ was changed from room temperature $21.0^{\circ} \mathrm{C}$ to $-15.0^{\circ} \mathrm{C}$. The definition of coordinates has been described in our previous studies $[16,17]$. In addition, the inclined angle of the surface, $\alpha$, is defined as the angle between the inclined surface and the horizontal plane. In the present study, five repeated tests were carried out for each case.

The water droplet impact process at an inclined angle of $0.0^{\circ}$ $\left(T_{w}=-10.0^{\circ} \mathrm{C}\right)$ is shown in Fig. 1 . The moment when the droplet impinged the surface was defined as the starting moment (Fig. 1a). Then, a lamella appeared immediately and the water droplet started to spread (Fig. 1b). Droplet extended to its maximum dimension and a pancake shape formed (Fig. 1e). The time duration of the spreading process was about $4.0 \mathrm{~ms}$ and the spreading maximum diameter was found to be $10.98 \mathrm{~mm}$. Then, the water droplet began to recoil (Fig. 1f). The water droplet reached its equilibrium state approximately at $t=1100.0 \mathrm{~ms}$. At last, the contact diameter of the stationary droplet was $5.61 \mathrm{~mm}$ (Fig. 11). It should be noted that the water droplet did not freeze during the entire impact process even though surface temperature was far below $0{ }^{\circ} \mathrm{C}$.

The water droplet impact process at an inclined angle of $30.0^{\circ}$ $\left(T_{w}=-10.0^{\circ} \mathrm{C}\right)$ is shown in Fig. 2 . Since the surface is inclined, gravity also plays an important role during the droplet impact process. The water droplet was found to go through five phases: kinetic phase, spreading phase, gliding phase, relaxation phase, and wetting phase. The time duration of the spreading process was about $3.0 \mathrm{~ms}$ and the spreading maximum diameter was found to be $9.17 \mathrm{~mm}$ (Fig. 2d). Then, the water droplet started to glide downward due to gravity and the foremost point moved faster than the rearmost point (Fig. 2e). The gliding phase ended at $t=8.5 \mathrm{~ms}$ and the gliding maximum diameter was $11.86 \mathrm{~mm}$ (Fig. 2f). After that, the water droplet began to recoil (Fig. 2g). The relaxation phase of the droplet continued until $t=1500.0 \mathrm{~ms}$, which was longer than that of the $\alpha=0.0^{\circ}$ case. At last, the contact diameter of the stationary droplet was $5.61 \mathrm{~mm}$ (Fig. 2l).

The water droplet impact process at an inclined angle of $45.0^{\circ}$ $\left(T_{w}=-10.0^{\circ} \mathrm{C}\right)$ is shown in Fig. 3. Since the surface was further inclined, gravity played a bigger role during the droplet impact process. The time duration of the spreading process was about $2.0 \mathrm{~ms}$ (Fig. 3e). Then, the water droplet started to glide downward

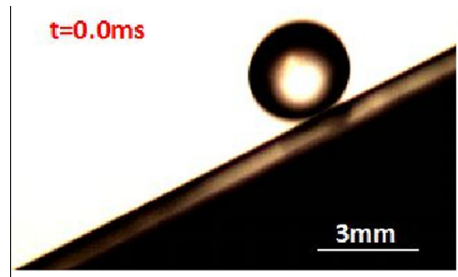

(a)

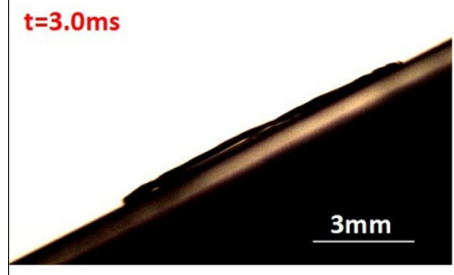

(d)

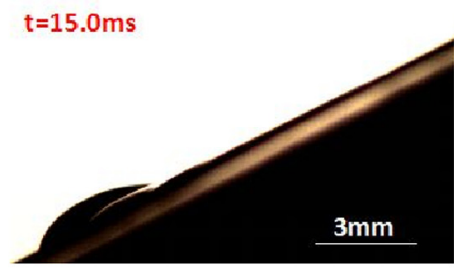

(g)

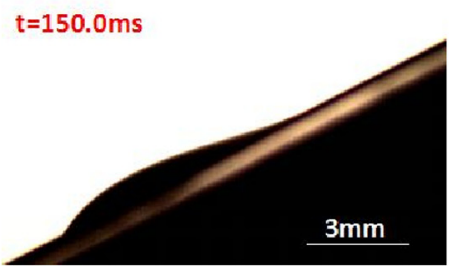

(j)

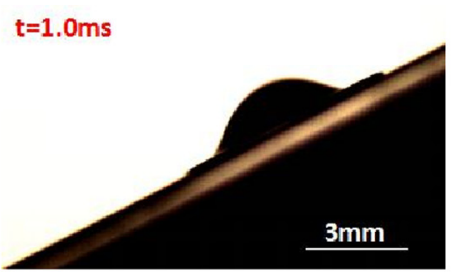

(b)

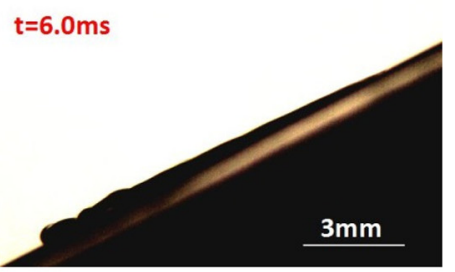

(e)

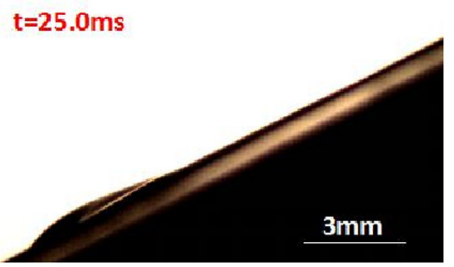

(h)

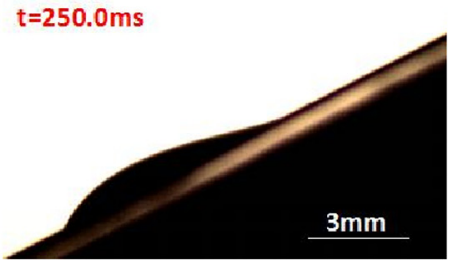

(k)

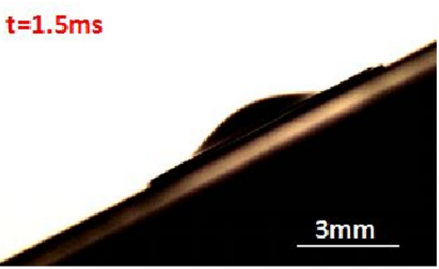

(c)

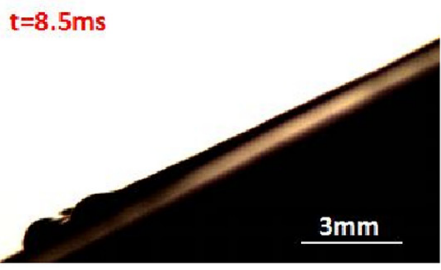

(f)

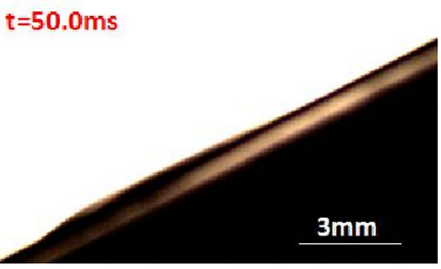

(i)

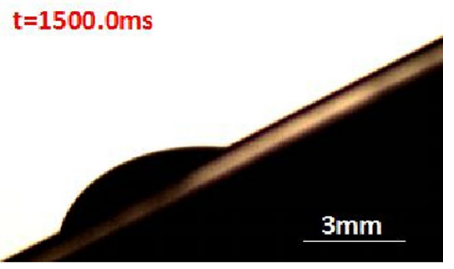

(1)

Fig. 2. Sequence of images of the water droplet impact process at an inclined angle of $30.0^{\circ}\left(T_{w}=-10.0^{\circ} \mathrm{C}\right)$. 


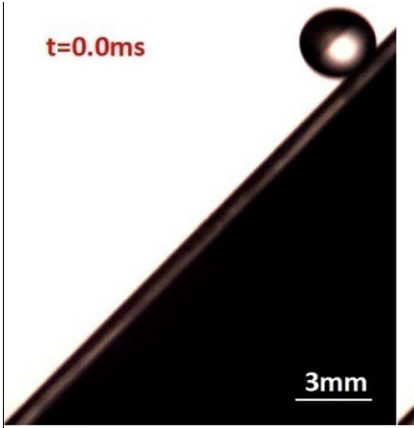

(a)

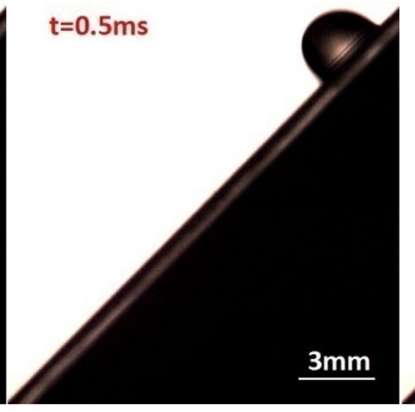

(b)

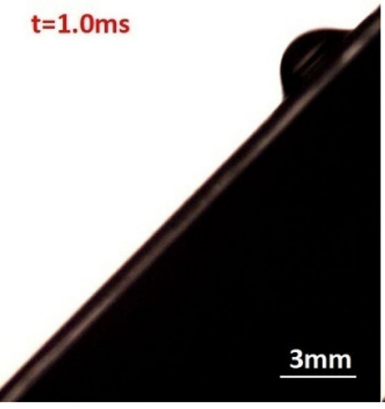

(c)

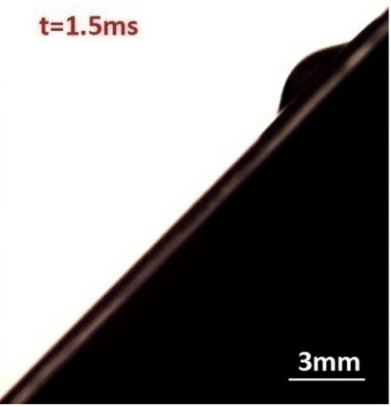

(d)

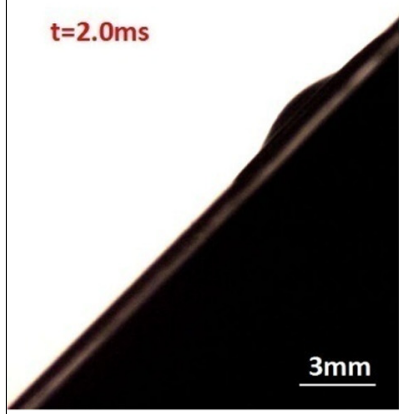

(e)

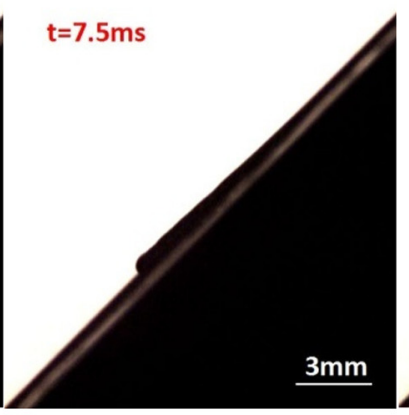

(f)

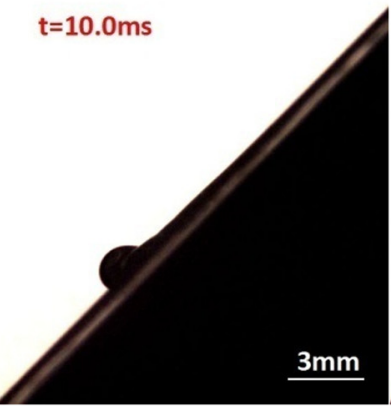

(g)

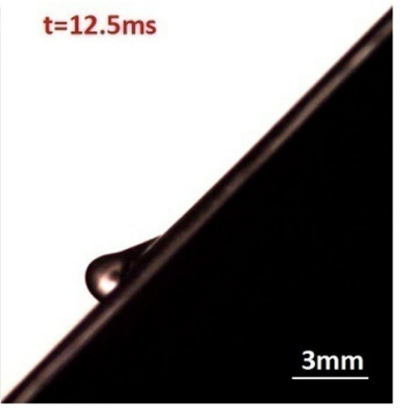

(h)

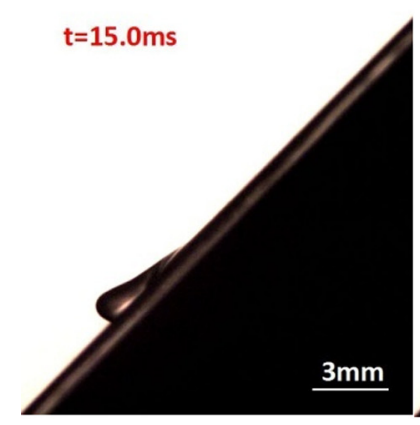

(i)

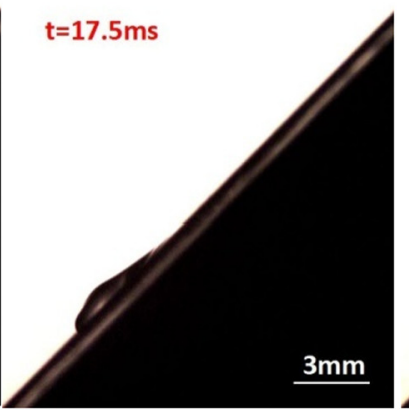

(j)

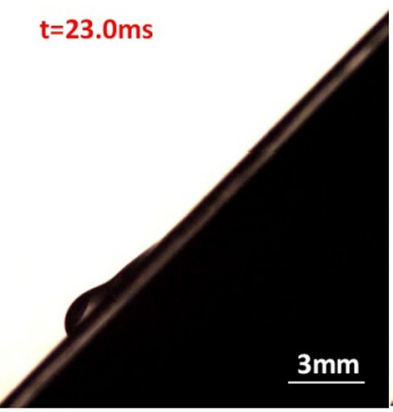

(k)

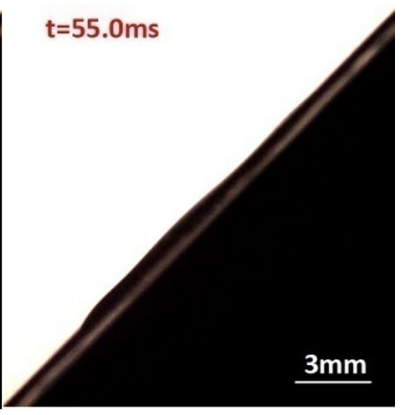

(1)

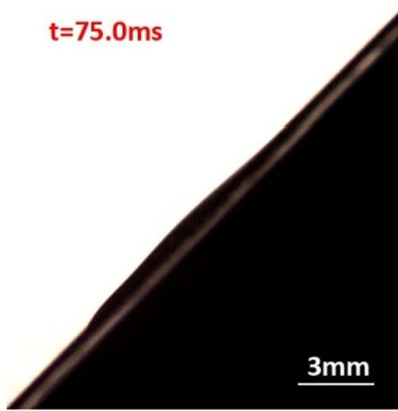

(m)

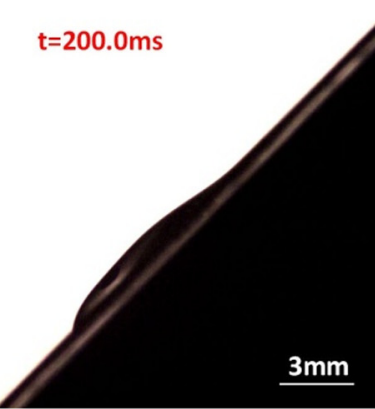

(n)

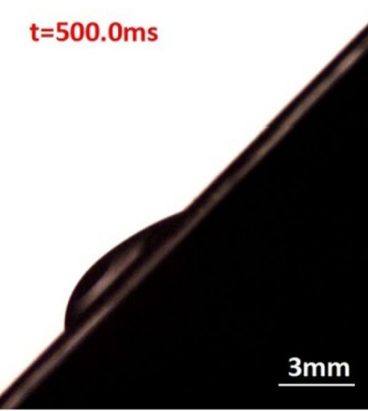

(o)

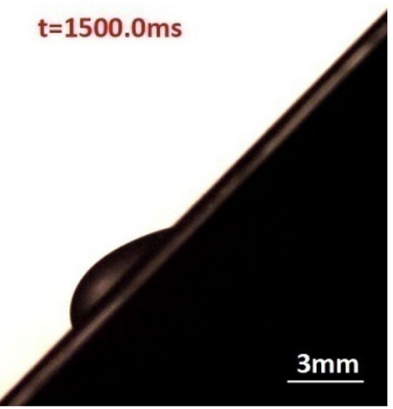

(p)

Fig. 3. Sequence of images of the water droplet impact process at an inclined angle of $45.0^{\circ}\left(T_{w}=-10.0^{\circ} \mathrm{C}\right)$.

(Fig. 3f). It appeared that water accumulated quickly near the foremost point and formed a relatively bulk water region (Fig. $3 \mathrm{~g}$ and h). Due to its large size and high speed, such a region could overcome the restriction of the surface tension, surpass the original foremost point, and fall onto the inclined surface (Fig. 3i and $\mathrm{j}$ ). Compared to the $\alpha=30.0^{\circ}$ case (Fig. 2), the higher inclined angle allowed the water droplet to have a larger momentum in the tangent direction at the early stage of the impact process. This tangent momentum together with gravity was believed to be the primary factor that increased the kinetic energy inside the bulk water region. Once the foremost point of the water droplet stopped moving downward (Fig. 3k), the surface tension caused the water droplet to recoil (Fig. 31, $\mathrm{m}$ and $\mathrm{n}$ ). At last, the contact diameter of the stationary droplet was $5.78 \mathrm{~mm}$ (Fig. 3p).

Fig. 4 shows the water droplet impact process at an inclined angle of $60.0^{\circ}\left(T_{w}=-10.0^{\circ} \mathrm{C}\right)$. In order to record the complete 


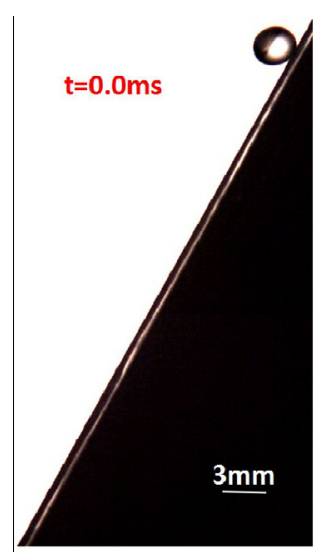

(a)

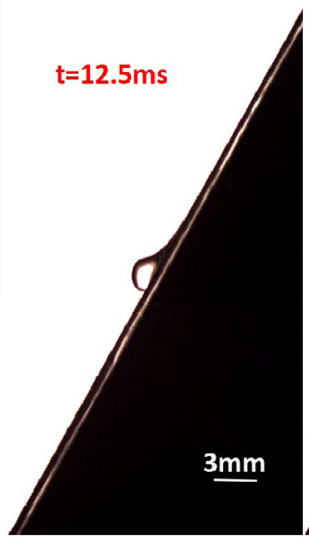

(e)

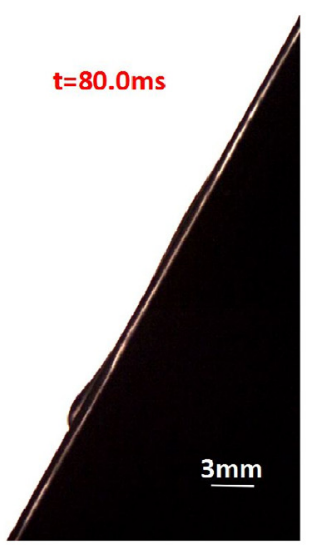

(i)

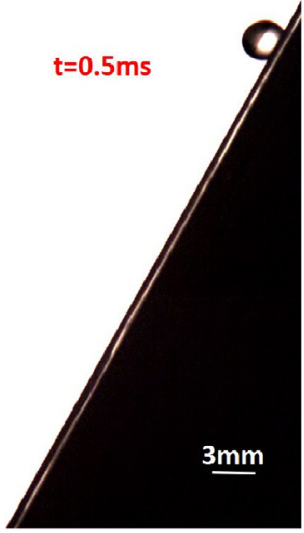

(b)

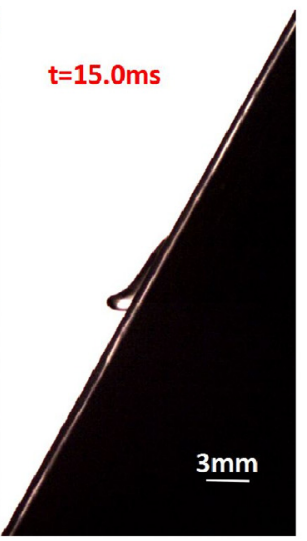

(f)

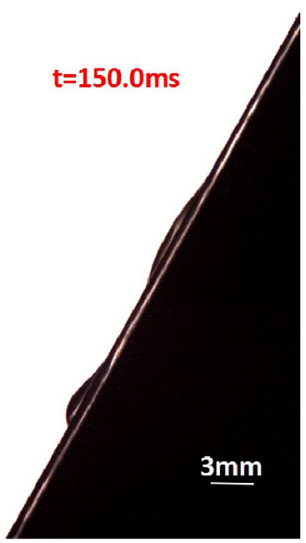

(j)

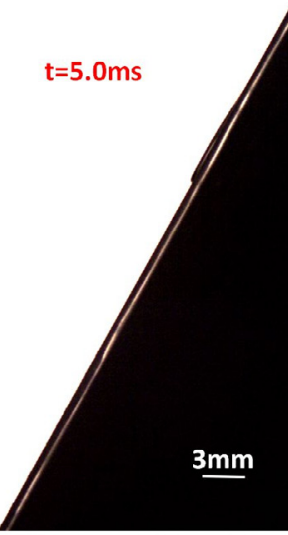

(c)

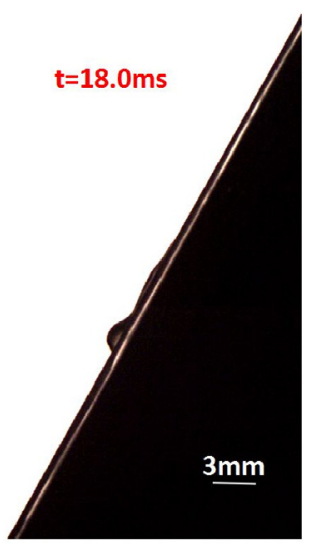

(g)

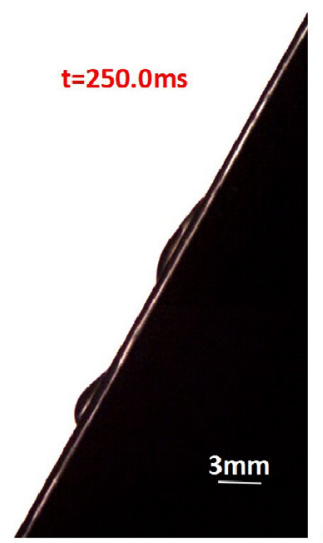

(k)

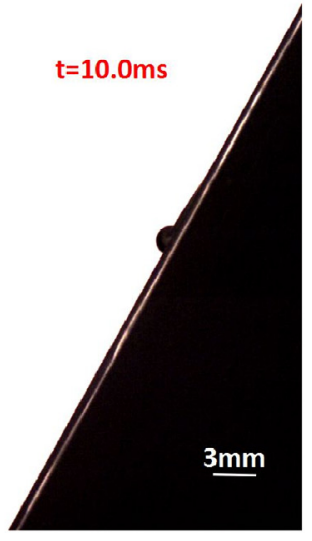

(d)

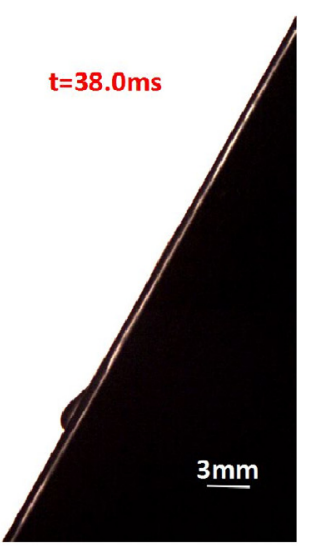

(h)

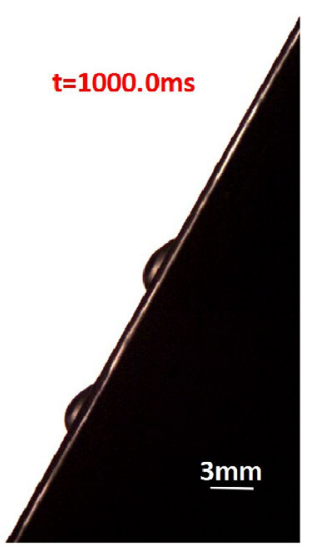

(1)

Fig. 4. Sequence of images of the water droplet impact process at an inclined angle of $60.0^{\circ}\left(T_{w}=-10.0^{\circ} \mathrm{C}\right)$.

impact process of the water droplet, the region of interest of the high-speed camera was enlarged. In this case, due to the relatively high inclined angle, the spreading process of the water droplet lasted for only about $0.5 \mathrm{~ms}$, which was indicated by that the rearmost point stopped moving upward (Fig. 4b). Then, the water droplet started to glide downward swiftly due to gravity (Fig. 4c). Similar to the $\alpha=45.0^{\circ}$ case, water started to accumulate quickly near the foremost point and formed a relatively bulk water region (Fig. $4 \mathrm{~d}$ and e). Such a region overcame the restriction of the surface tension, surpassed the original foremost point, and fell onto the inclined surface (Fig. $4 \mathrm{f}$ and $\mathrm{g}$ ). Then, the foremost point of the water droplet kept moving downward for a certain distance before it stopped (Fig. $4 \mathrm{~h}$ ). After that, the water droplet started to recoil. However, instead of remaining as a single water droplet, the water accumulated at two different locations and finally two smaller droplets appeared (Fig. 4j, k, and 1). In order to better explain this matter, the water droplet formed further downward was named as the lower droplet, while the other one was defined as the upper droplet. It should be noted that a satellite droplet appeared in between the upper and lower droplets. Due to its small size, the satellite droplet is difficult to observe in Fig. 4 and will be illustrated in Fig. 5.

In order to have a better insight of the split phenomenon when the water droplet impinged onto the surface at an inclined angle of $60.0^{\circ}$, a high-speed camera (FR180, Norpix) was utilized to take images from a perspective perpendicular to that of the high speed 


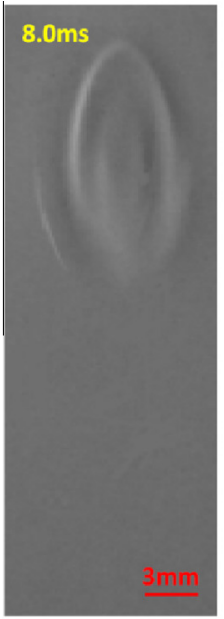

(a)

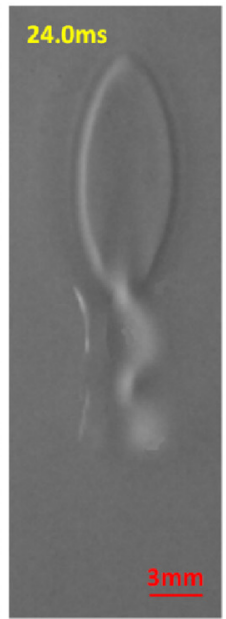

(b)

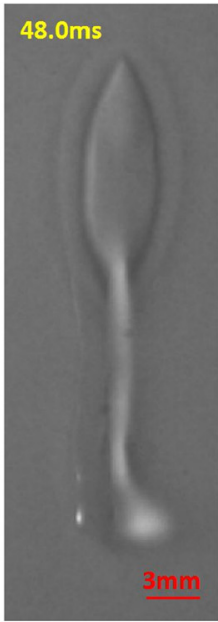

(c)

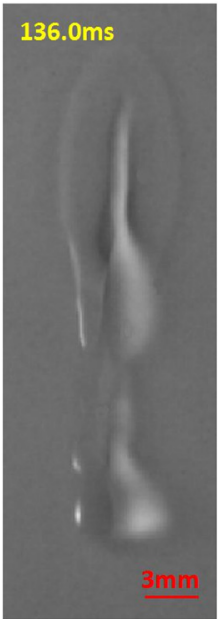

(d)

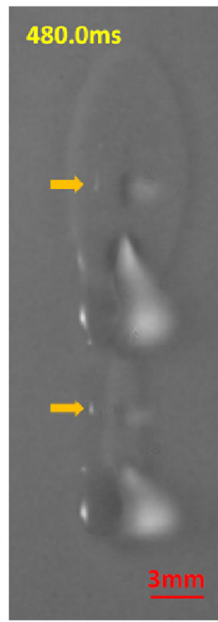

(e)

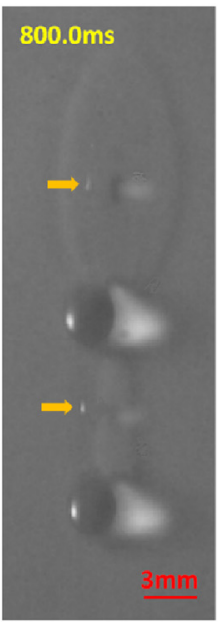

(f)

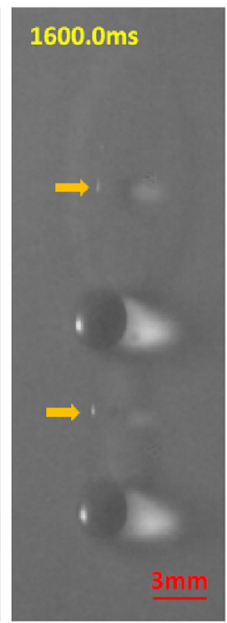

(g)

Fig. 5. Sequence of images of the water droplet impact process at an inclined angle of $60.0^{\circ}\left(T_{w}=-5.0^{\circ} \mathrm{C}\right)$.

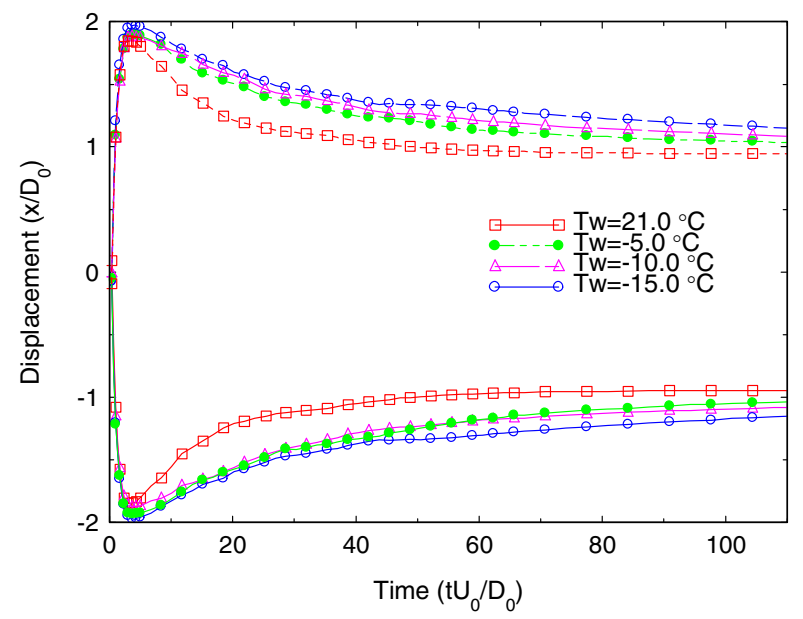

(a) $0.0^{\circ}$

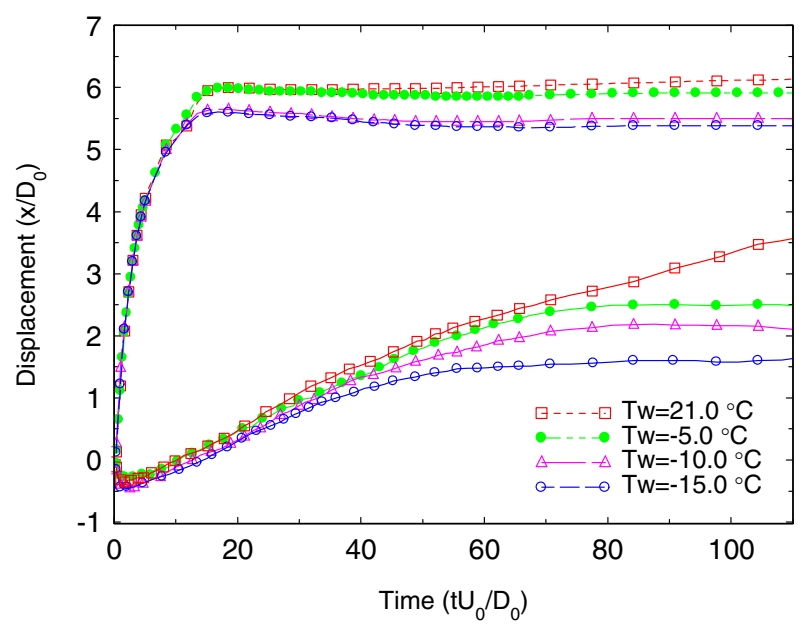

(c) $45.0^{\circ}$

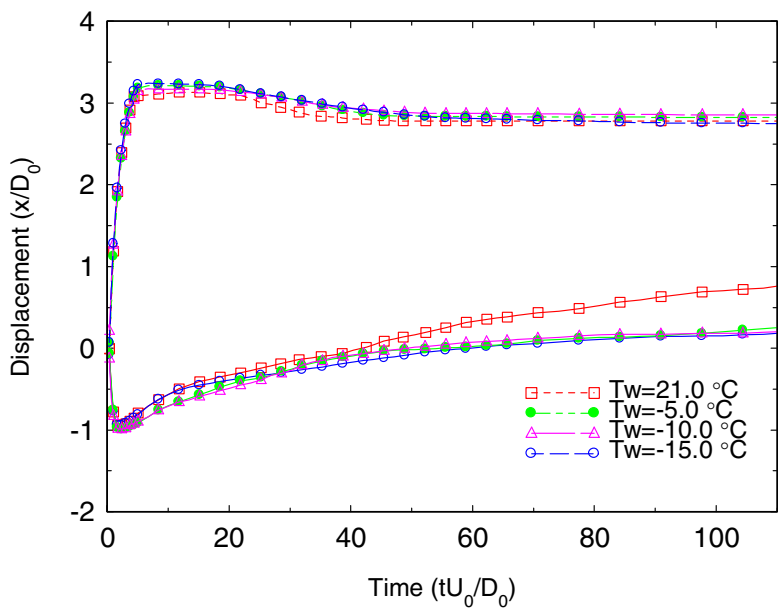

(b) $30.0^{\circ}$

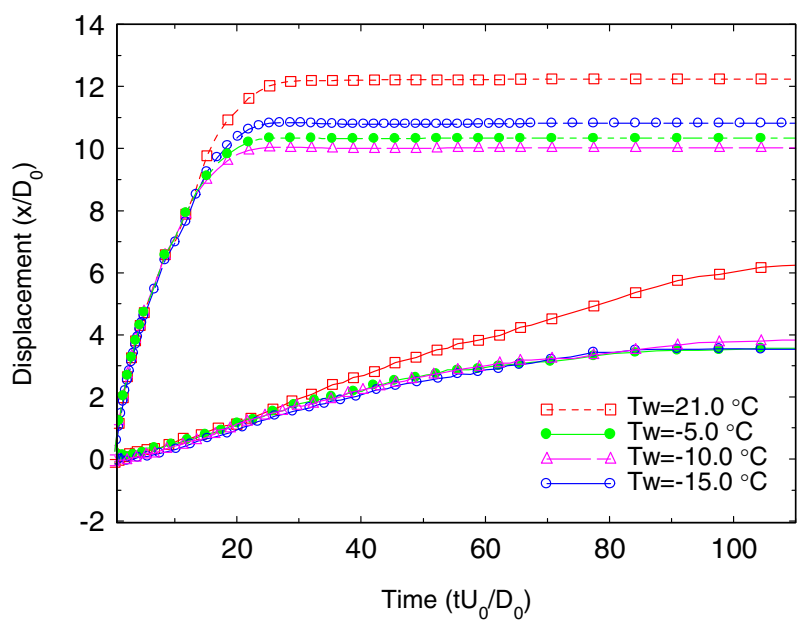

(d) $60.0^{\circ}$

Fig. 6. The change of displacements of foremost point (dashed line) and rearmost point (solid line) with time.

camera (Dimax HD, PCO), which was operated at $125 \mathrm{~Hz}$. Fig. 5 shows the sequence of the images of the water droplet impact pro- cess $\left(T_{w}=-5.0^{\circ} \mathrm{C}\right)$ obtained by this camera. Thus, the whole split phenomenon of the water droplet can be explained as follows: 
Table 1

Comparison of the ice beads at different inclined angles.

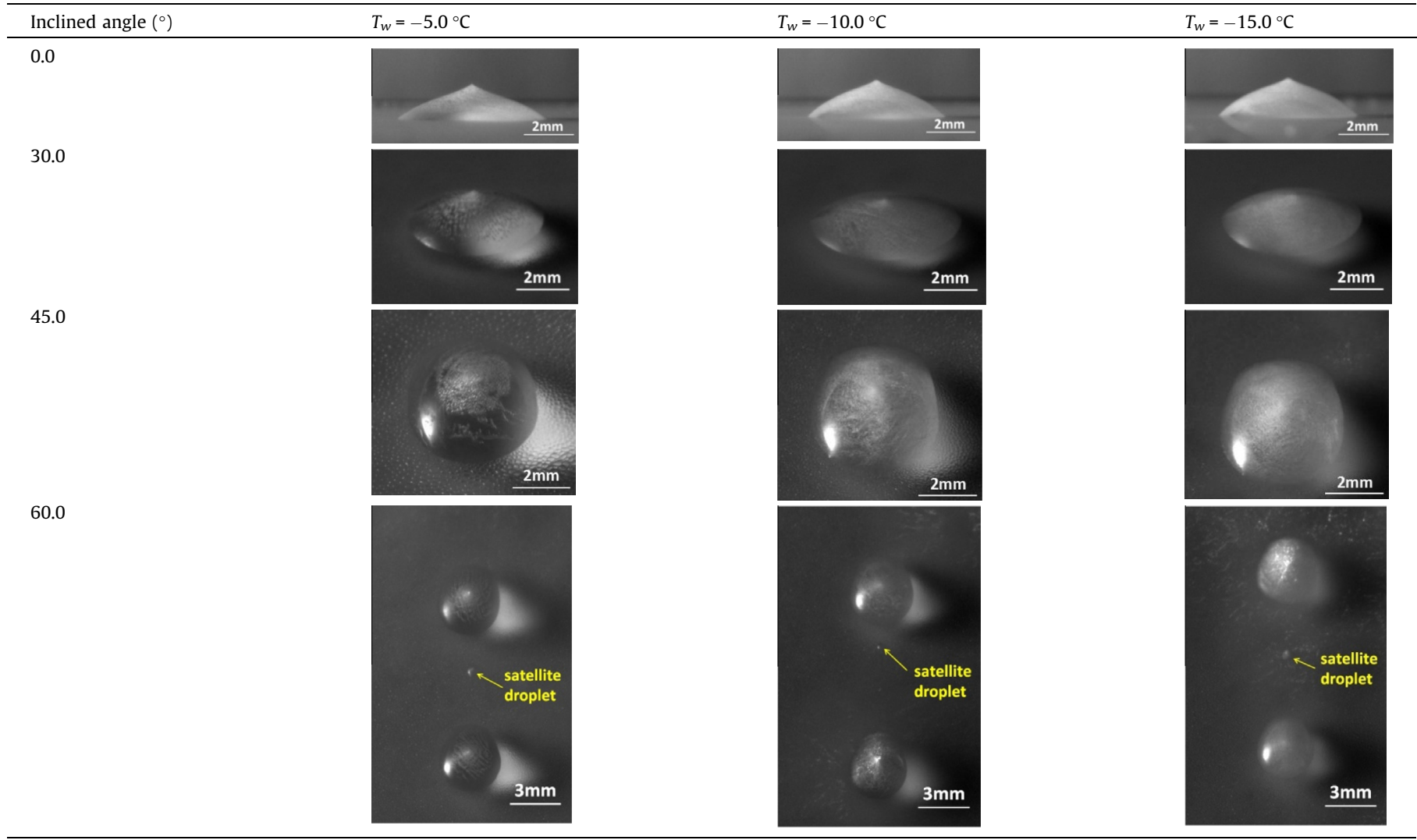

Firstly, during the early stage of the impact process of the water droplet, the initial water droplet extended to a thin water film which occupied a large area (Fig. 5a); Secondly, water started to accumulate quickly near the foremost point and formed a relatively bulk water region. Then, such a bulk water region moved downward rapidly (Fig. 5b); Thirdly, once the droplet stopped moving downward (Fig. 5c), it started to recoil; Fourthly, the initial water film broke in the middle and water started to concentrated towards two regions (Fig. 5d); Finally, the upper and lower droplets as well as two satellite droplets were generated gradually (Fig. 5e and f). One satellite droplet was located above the upper droplet and the other one appeared between the upper and lower droplets (Fig. 5e and f). In Fig. 5, the locations of the satellite droplets were specified by yellow arrows.

Fig. 6 demonstrates the change of the displacements of foremost and rearmost points of the droplet with time at different inclined angles. The results were based on the average of five measurements. Both time and displacement were normalized by following the previous studies $[14,18]$. At $\alpha=0.0^{\circ}$, since the surface was horizontal, the foremost and rearmost points actually corresponded to the leftmost and rightmost points of the water droplet (Fig. 6a). The movements of the leftmost and rightmost points were symmetrical with respect to the impact point. The decrease of surface temperature resulted in a slower retraction of the leftmost and rightmost points during the relaxition process. Besides, the maximum displacements of the leftmost and rightmost points of the water droplet were found to be less than 2. As for the $\alpha=30.0^{\circ}$ case, the movements of the leftmost and rightmost points were no longer symmetrical with respect to the impact point (Fig. 6b). The change of the surface temperature had a minor influence on the movement of the foremost point. However, the rearmost point was found to retract faster at room temperature than that of the other three cases. The minimum displacements of the rearmost point of the water droplets were about -1 . At $\alpha=45.0^{\circ}$, the displacements of the foremost and rearmost points appeared to be much larger than those of the $\alpha=0^{\circ}$ and $30.0^{\circ}$ cases (Fig. 6c). Moreover, the effect of the surface temperature on the displacement of the rearmost point was apparent. In addition, the minimum displacements of the rearmost point of the water droplets were about -0.5 while the maximum displacements of the foremost point of the water droplets were larger than 5 . As for the $\alpha=60.0^{\circ}$ case (Fig. $6 \mathrm{~d}$ ), since the initial water droplet split into two smaller droplets, the foremost point was actually taken from the lower droplet while the rearmost point was extracted from the upper droplet. Large discrepancies presented between the displacements of the foremost and rearmost points at room temperature and those of the other cases. In this case, the minimum displacements of the rearmost point of the water droplets were about -0.2 while the maximum displacements of the foremost point of the water droplets were all larger than 9 .

Table 1 illustrated the images of the ice beads at the end of the droplet freezing process. As for the $\alpha=60.0^{\circ}$ case, since the initial water droplet broke into upper droplet, lower droplet, and two satellite droplets (Fig. 5), the ice beads for this case were totally different from those of the other cases. At lower inclined angles (e.g., $\alpha=0.0^{\circ}, 30.0^{\circ}$, and $45.0^{\circ}$ ), the inclined angle of the surface had an apparent influence on the final shape of the ice bead. Such a phenomenon could be explained as follows: When a water droplet is placed on an ideal horizontal surface, its shape is axisymmetric, so that the contact line is circular and the contact angle distribution along the contact line is uniform. However, once a water droplet is placed on an inclined surface, gravity is applied to such a resting droplet. Thus, the contact angle distribution along the contact line must distort in order to resist the droplet motion 
[19]. The differences in the geometries of the sessile water droplets result in the variations of the shape of the ice bead eventually.

\section{Conclusions}

In summary, we experimentally investigated the impact and freezing processes of a water droplet on a cold surface with different inclined angles. Our results demonstrated that the inclined angle of the surface had a significant effect on the impact process of the water droplet. As the increase of the inclined angle, gravity played a bigger role on the droplet kinematics and the water droplet also had a larger tangent momentum at the early stage of the impact process. These factors directly influenced the spreading time and the displacements of the foremost and rearmost points of the water droplet. Once the inclined angle of the surface was high enough (e.g., $\alpha=60.0^{\circ}$ ), the water droplet could not keep as a single droplet all the time and split into smaller droplets during its impact process. Besides, the inclined angle also affected the contact angle distribution along the contact line of the sessile droplet. As for the freezing process, the inclined angle of the cold surface affected the shape of the ice beads.

\section{Acknowledgments}

This work was supported by Science and Technology Commission of Shanghai Municipality (Grant No.: 15ZR1442700), Fundamental Research Funds for the Central Universities, and Opening fund of State Key Laboratory of Nonlinear Mechanics.

\section{References}

[1] N. Dalili, A. Edrisy, R. Carriveau, A review of surface engineering issues critical to wind turbine performance, Renew. Sustain. Energy Rev. 13 (2009) 428-438.

[2] Civil Aviation Authority of New Zealand, Aircraft Icing Handbook, Civil Aviation Authority (CAA), 2000.
[3] J.L. Laforte, M.A. Allaire, J. Laflamme, State-of-the-art on power line de-icing, Atmos. Res. 46 (1998) 143-158.

[4] B.S. Kang, D.H. Lee, On the dynamic behavior of a liquid droplet impacting upon an inclined heated surface, Exp. Fluids 29 (2000) 380-387.

[5] Š. Šikalo, C. Tropea, E.N. Ganić, Impact of droplets onto inclined surfaces, J. Colloid Interf. Sci 286 (2005) 661-669.

[6] Š. Šikalo, E.N. Ganić, Phenomena of droplet-surface interactions, Exp. Therm. Fluid. Sci 31 (2006) 97-110.

[7] J. Cui, X. Chen, F. Wang, X. Gong, Z. Yu, Study of liquid droplets impact on dry inclined surface, Asia-Pac. J. Chem. Eng. 4 (2009) 643-648.

[8] L. Mishchenko, B. Hatton, V. Bahadur, J.A. Taylor, T. Krupenkin, J. Aizenberg, Design of ice-free nanostructured surfaces based on repulsion of impacting water droplets, ACS Nano 4 (2010) 7699-7707.

[9] Y. Ueda, Y. Sakai, T. Kumagai, T. Ishii, M. Iguchi, A bouncing droplet on an inclined hydrophobic surface, J. Visual. 13 (2010) 5-6.

[10] Q. Xu, Z. Li, J. Wang, R. Wang, Characteristics of single droplet impact on cold plate surfaces, Dry. Technol. 30 (2012) 1756-1762.

[11] T. Gilet, J.W.M. Bush, Droplets bouncing on a wet, inclined surface, Phys. Fluids 24 (2012) 122103

[12] Y. Wang, J. Xue, Q. Wang, Q. Chen, J. Ding, Verification of icephobic/anti-icing properties of a superhydrophobic surface, ACS Appl. Mater. Interf. 5 (2013) 3370-3381.

[13] S. Quan, W. Li, S. Shen, Y. Guo, Simulation of droplet impact onto horizontal and inclined solid surfaces with Lattice-Boltzmann method, J. Harbin Inst. Technol. 20 (2013) 97-105.

[14] G. Liang, Y. Guo, Y. Yang, N. Zhen, S. Shen, Spreading and splashing during a single drop impact on an inclined wetted surface, Acta Mech. 224 (2013) 2993-3004.

[15] R. Ramachandran, K. Sobolev, M. Nosonovsky, Dynamics of droplet impact on hydrophobic/icephobic concrete with the potential for superhydrophobicity, Langmuir 31 (2015) 1437-1444.

[16] Z. Jin, D. Sui, Z. Yang, The impact, freezing, and melting processes of a water droplet on an inclined cold surface, Int. J. Heat. Mass. Transfer 90 (2015) 439453.

[17] Z. Jin, Z. Wang, D. Sui, Z. Yang, The impact and freezing processes of a water droplet on different inclined cold surfaces, Int. J. Heat. Mass. Transfer 97 (2016) 211-213.

[18] Š. Šikalo, M. Marengo, C. Tropea, E.N. Ganic, Analysis of impact of droplets on horizontal surfaces, Exp. Therm. Fluid Sci 25 (2002) 503-510.

[19] C. Antonini, FJ. Carmona, E. Pierce, M. Marengo, A. Amirfazli, General methodology for evaluating the adhesion force of drops and bubbles on solid surfaces, Langmuir 25 (2009) 6143-6154. 